\title{
Pyruvate production using engineered Escherichia coli
}

\author{
Hironaga Akita ${ }^{1}$, Nobutaka Nakashima ${ }^{2,3^{*}}$ and Tamotsu Hoshino ${ }^{1,2}$
}

\begin{abstract}
Pyruvate plays an essential role in the central carbon metabolism of multiple organisms and is used as a raw material in the chemical, biochemical and pharmaceutical industries. To meet demand, large amounts of pyruvate are produced through fermentation processes. Here we describe a simple and efficient method for producing pyruvate in Escherichia coli. To stop carbon flux from pyruvate to fatty acids, the accBC genes, which encode the enzyme that catalyzes the first step of fatty acid biosynthesis and is essential for vegetative growth, were manipulated within the genome; its native promoter was replaced with the tetracycline (or doxycycline)-regulated promoter and the corresponding transcriptional regulator genes. The resulting strain grew normally in the presence of doxycycline, but showed poor growth upon withdrawal of doxycycline. Using this strain, we developed a high pyruvate producing

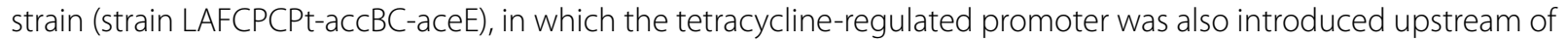
aceE, and the ackA-pta, adhE, cra, IdhA, pflB and poxB genes were deleted. After determining the optimal culture conditions for this strain, the final pyruvate concentration reached $26.1 \mathrm{~g} \mathrm{~L}^{-1}$ after $72 \mathrm{~h}$ with a theoretical yield of $55.6 \%$. These levels are high enough to indicate that the developed strain has the potential for application to industrial production of pyruvate.
\end{abstract}

Keywords: Pyruvate, Escherichia coli, Promoter regulation, Tetracycline, Doxycycline, Fermenter

\section{Introduction}

Pyruvate is a key metabolite in such catabolic and anabolic pathways as glycolysis, gluconeogenesis, and amino and fatty acid metabolism. In addition, commercial demand for pyruvate has been expanding every year. In biochemical industries, for example, pyruvate is used as a building block for the synthesis of antioxidants (Wang et al. 2007), food additives (EFSA 2009), and dietary and weight control supplements (Kalman et al. 1999; Saper et al. 2004). Pyruvate is also used as a raw material in the pharmaceutical industry. For example, various pharmaceutical precursors, including $N$-acetyl-D-neuraminic acid (Zhang et al. 2010), L-3,4-dihydroxyphenylalanine (Park et al. 1998) and R-phenylacetylcarbiol (Rosche et al. 2001) are produced from pyruvate.

\footnotetext{
*Correspondence: nnakashima@bio.titech.ac.jp

${ }^{3}$ Department of Biological Information, Graduate School of Bioscience and Biotechnology, Tokyo Institute of Technology, 2-12-1-M6-5 Ookayama, Meguro-ku, Tokyo 152-8550, Japan

Full list of author information is available at the end of the article
}

Commercial methods of pyruvate production can be roughly classified as chemical, enzymatic or fermentation processes ( $\mathrm{Li}$ et al. 2001a). In the chemical process, pyruvate is synthesized mainly through dehydration and decarboxylation of tartrate. But while this method is easy to implement, it is not cost effective ( $\mathrm{Li}$ et al. 2001a). Compared with the chemical process, the enzymatic process effectively reduces the production cost and improves yield ( $\mathrm{Li}$ et al. 2001a). The fermentation process is the most widely used in industry, because it is sustainable, cost-effective and achieves high yields and productivity (Li et al. 2001a; Xu et al. 2008). For the fermentation process, a multi-vitamin auxotroph strain of Torulopsis glabrata is primarily used ( $\mathrm{Li}$ et al. 2001a). With T. glabrata strains, a pyruvate concentration of more than $40 \mathrm{~g} \mathrm{~L}^{-1}$ was reached (Li et al. 2001a), though maintenance of the concentration balance among biotin, nicotinic acid, pyridoxine and thiamine was required ( $\mathrm{Li}$ et al. 2001b). Consequently, the availability of this method is limited by the necessity for special expertise and expensive equipment. On the other hand, 
a lipoic acid auxotroph strain of Escherichia coli has also developed as a producer (Table 1). For example, $E$. coli CGSG 7916 showed high pyruvate accumulation in controlled fermentations (Tomar et al. 2003). This strain was constructed through aceF mutation. The aceEF and lpd genes express the pyruvate dehydrogenase complex, which catalyzes conversion of pyruvate into acetyl-CoA (Fig. 1; CaJacob et al. 1985). Thus, pyruvate accumulation was achieved by decreasing the activity of the pyruvate dehydrogenase complex. In addition, E. coli YYC202, an aceEF deletion strain, showed excellent pyruvate productivity (Zelić et al. 2003). However, this strain required acetate for growth due to its weak capacity for acetylCoA production. E. coli TBLA-1 was constructed by transduction of a F1-ATPase-defective gene into E. coli W1485lip2, which is a derivative strain from $E$. coli $\mathrm{K}-12$ (Yokota et al. 1994). This mutation enhanced both glucose consumption and pyruvate production. E. coli TC44 was constructed through decreasing the ATP yield, cell growth and $\mathrm{CO}_{2}$ production as well as deletion of the acetate, ethanol and lactate production pathways (Causey et al. 2004). During E. coli TC44 fermentations, changing the oxygen saturation from 5 to $50 \%$ enhanced the pyruvate productivity. In E. coli TC44, mutation of the poxB gene, which encodes pyruvate oxidase, was most beneficial for growth and pyruvate production, as the mutation enhanced the $\mathrm{NAD}^{+}$concentration in the cells and activated several enzymes involved in the glycolysis pathway. Thus construction of E. coli strains for pyruvate production involves introducing mutations that reduce utilization of pyruvate for cell growth and deletion of nonessential pathways through pyruvate metabolism.

Here we report an effective method for using promoter regulation for pyruvate production. To control essential gene expressions, tetracycline-regulated promoter (Ptet) was inserted upstream of the $a c c B C$ genes, and gene expressions were regulated by the absence of doxycycline. After the pyruvate producing strain was constructed, optimal culture conditions for pyruvate production were determined.

Table 1 Comparison of the pyruvate productivities of $E$. coli strains

\begin{tabular}{|c|c|c|c|c|c|c|}
\hline Strain & Genotype & Carbon source & $\begin{array}{l}\text { Concentrations } \\
\left(\mathrm{g} \mathrm{L}^{-1}\right)\end{array}$ & $\begin{array}{l}\text { Productivity } \\
\left(g^{-1} h^{-1}\right)\end{array}$ & Yield $^{a}(\%)$ & References \\
\hline $\begin{array}{l}\text { E. coli LAFCPCPt-accBC- } \\
\text { aceE }\end{array}$ & $\begin{array}{c}\text { MG(1655) } \triangle \text { ldhA } \triangle a d h E \\
\triangle p f l B \triangle p t a-a c k A \\
\triangle p o x B \triangle c r a \text { Ptet- } \\
\text { accBC Ptet-aceE }\end{array}$ & Glucose & $4.2^{b}$ & 0.0584 & 10.6 & This study \\
\hline $\begin{array}{l}\text { E. coli LAFCPCPt-accBC- } \\
\text { aceE }\end{array}$ & $\begin{array}{c}\text { MG(1655) } \triangle l d h A \triangle a d h E \\
\triangle p f l B \triangle p t a-a c k A \\
\triangle p o x B \triangle c r a \text { Ptet- } \\
\text { accBC Ptet-aceE }\end{array}$ & Glucose & $26.1^{c}$ & 0.363 & 55.6 & This study \\
\hline E. coli CGSG (6162) & $\begin{array}{l}\mathrm{F}^{+} \text {aceF10 fadR200 } \\
\text { tyrT58(AS) adhE80 } \\
\text { mel-1 }\end{array}$ & Glucose, acetate & 37.0 & 1.03 & ND & Tomar et al. (2003) \\
\hline E. coli CGSG (7916) & CGSC(6162) ppc::Kan & Glucose, acetate & 35.0 & 0.972 & ND & Tomar et al. (2003) \\
\hline E. coliTC44 & $\begin{array}{l}\text { W(3110) }\left(\text { Succ }^{+}\right), \\
\triangle \text { focA-pflB::FRT } \\
\triangle \text { frdBC } \triangle / d h A \\
\triangle a t p(F H):: F R T \\
\triangle a d h E:: F R T \\
\triangle \text { sucA::FRT } \triangle p o \times B:: F R T \\
\triangle a c k A: \because F R T\end{array}$ & Glucose & 52.0 & 1.21 & 77.9 & Causey et al. (2004) \\
\hline E. coliW(1485)lip2 & $W(1485) \mathrm{F}^{+} \lambda^{-}$lipA2 & Glucose & 25.5 & 0.797 & 52.2 & Yokota et al. (1994) \\
\hline E. coli TBLA-1 & $\begin{array}{l}\text { W(1485)/ip2 bg/ }{ }^{+} \\
\text {atpA401 }\end{array}$ & Glucose & 31.5 & 0.984 & 64.4 & Yokota et al. (1994) \\
\hline E. coli YYC202 & $\begin{array}{l}\text { Hfr zbi::Tn } 10 \text { poxB1 } \\
\quad \triangle(\text { aceEF }) \text { rpsL pps-4 } \\
\text { pfl-1 }\end{array}$ & Glucose, acetate & 62.0 & 1.75 & ND & Zelić et al. (2003) \\
\hline E. coli ALS929 & YYC202 IdhA::Kan & Glucose, acetate & 70.0 & 2.06 & ND & Zhu et al. (2008) \\
\hline E. coli ALS(1059) & $\begin{array}{l}\text { YYC202 IdhA::Kan } \\
\text { arcA726::FRT } \\
\text { atpFH::Cam }\end{array}$ & Glucose, acetate & 90 & 2.05 & ND & Zhu et al. (2008) \\
\hline
\end{tabular}

ND not described

a Yield was the theoretical maximum value

b Pyruvate was produced from N5G medium under standard culture conditions for $72 \mathrm{~h}$

c Pyruvate was produced from N5G medium under optimized culture conditions for $72 \mathrm{~h}$ 


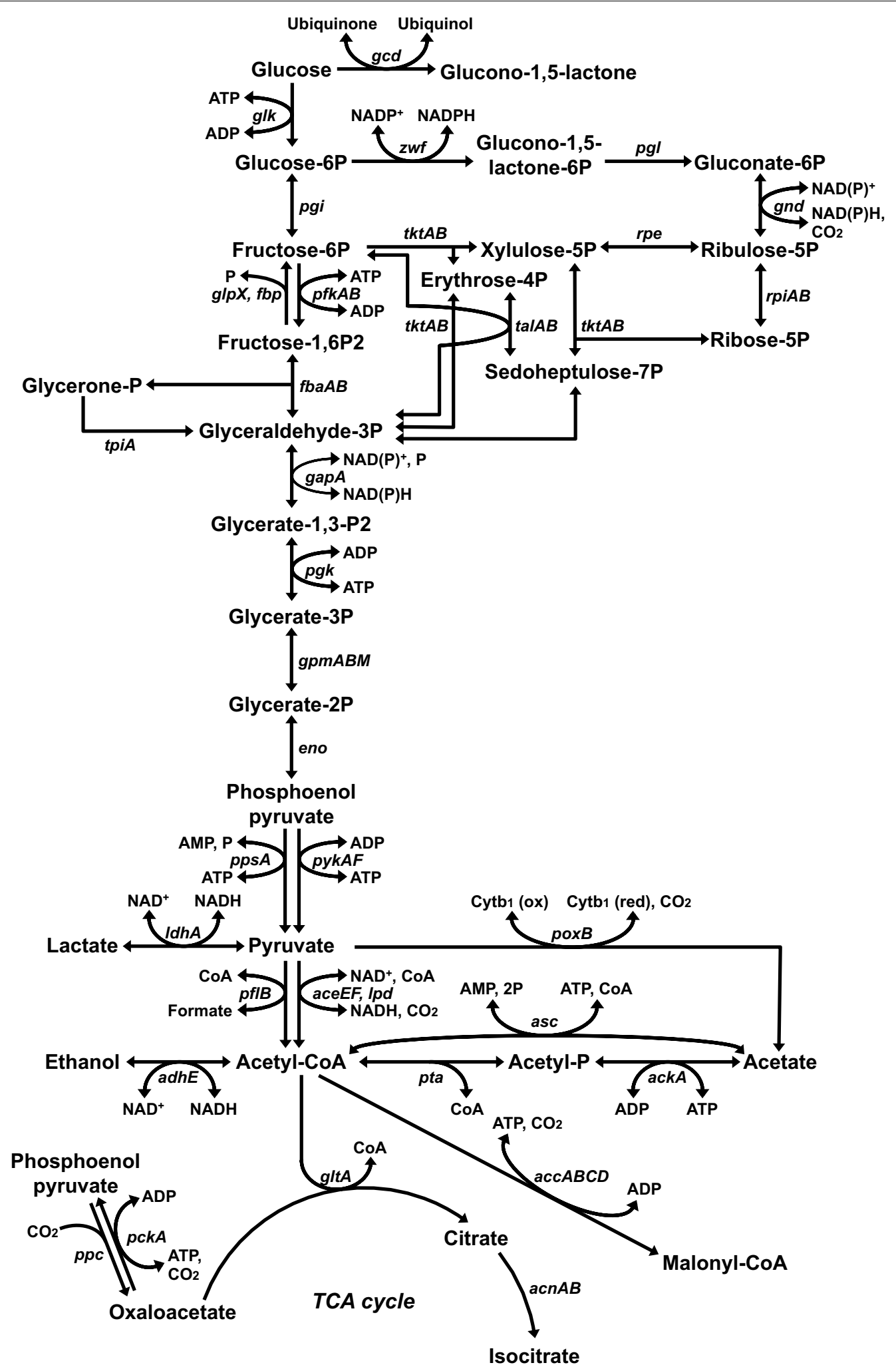

Fig. 1 Glucose metabolism in Escherichia coli 


\section{Materials and methods}

\section{General genetic techniques}

E. coli strain MG1655 (wild-type, The Coli Genetic Stock Center; CGSG6300) and its derivative strain LAF was described earlier (Nakashima and Tamura 2012). Gene deletion and knock-in were carried out as described earlier (Emmerson et al. 2006; Nakashima et al. 2014a, b; Nakashima and Miyazaki 2014). The sequences of all primers are shown in Table 2.

Unless stated otherwise, $E$. coli cells were cultured in Luria-Bertani medium at $37^{\circ} \mathrm{C}$. To evaluate cell growth, culture pre-grown overnight was diluted 1:500, and doxycycline was added if necessary. Cell density was measured at $600 \mathrm{~nm}$ in $200 \mu \mathrm{L}$ aliquots of culture in a 96-well plate (Nalge Nunc International, Rochester, NY, USA; product no. 269,620) using a Safire microplate reader (Tecan, Männedorf, Switzerland) and a LS-PLATEmanager 2004 data analysis program (Wako, Osaka, Japan).

\section{Construction of a plasmid for deletion of pox $B$}

The $5^{\prime}$ - and $3^{\prime}$-flanking regions of poxB were PCR-amplified from genomic DNA from strain MG1655 using specific primer sets: sSN1185/sSN1186 and sSN1187/ sSN1188, respectively. The $5^{\prime}$ end of sSN1185 was phosphorylated using T4 DNA kinase before use. The termini

Table 2 PCR primers for construction of plasmids

\begin{tabular}{|c|c|}
\hline Primers & Sequences \\
\hline sSN1185 & 5'-ATCCATGGTCGTCTCACTCGCGAACTGC-3' \\
\hline sSN1186 & 5'-GTGGATCCCCAGATGCGTTTCACCCCTGC-3' \\
\hline sSN1187 & 5'-AATCTAGAACAGGCCAAAGGTTTCAGCCTG-3' \\
\hline sSN1188 & 5'-GATATGCATCGCCACTTTATCCAGCGGTAG-3' \\
\hline sSN1892-accN & $\begin{array}{l}\text { 5'-AAACTGCAGGGGTGAATAATCTGATTTTGTTTGACT } \\
\text { AC-3' }\end{array}$ \\
\hline sSN1893-accN & $\begin{array}{l}\text { 5'-AAATCTAGACAGGCGTTTCACCGCCGTCTGTAAA } \\
\text { GC-3' }\end{array}$ \\
\hline sSN1894-tetR & 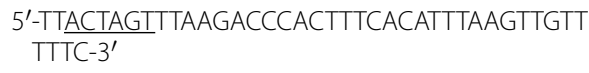 \\
\hline sSN1895-tetR & $\begin{array}{l}\text { 5'-AAAGGATCCTTTCTCCTCTTTAATGAATTCGGTCAG } \\
\text { TG-3' }\end{array}$ \\
\hline sSN1896-accORF & $\begin{array}{l}\text { 5'-AAAGGATCCATGGATATTCGTAAGATTAAAAAACT } \\
\text { G-3' }\end{array}$ \\
\hline sSN1897-accORF & 5'-CCGACATGTTCAGATAACTTTTTACTGACGGAGC-3' \\
\hline sSN1997aceEDox1 & 5'-TCAACATGTCTAACTACTTCAATACACTGAATCTG-3' \\
\hline sSN1998aceEDox2 & $\begin{array}{l}\text { 5'-AGACTAGTTTAACCCCCCAGTTTCGATTTATCGC } \\
\text { G-3' }\end{array}$ \\
\hline sSN1998aceEDox3 & $\begin{array}{l}\text { 5'-AAAATGCATAGGCCTTCTCGGGCATAAGTCGGACA } \\
\text { CCATGG-3' }\end{array}$ \\
\hline sSN1999aceEDox4 & 5'-ATTGCGAGGCTTTGTGCTTCTCTGG-3' \\
\hline sSN2000aceEDox5 & 5'-CCCATGGGGATCCAGGCCTTCTAGAACT-3' \\
\hline sSN2001aceEDox6 & 5'-GGGGTACCTGAGCAAACTGGCCTCA-3' \\
\hline
\end{tabular}

The restriction enzyme site is underlined of the amplified fragments were treated with $\mathrm{NcoI}$ and $N s i$, respectively, and the two fragments were cloned into the PstI-NcoI site of pHN1234, yielding pHN1243. Plasmid pHN1243 was used for disruption of poxB.

\section{Construction of plasmids for knock-in of tetracycline promoters}

A DNA fragment containing the $5^{\prime}$-flanking region of $a c c B C$ was PCR-amplified using primers sSN1892-accN and sSN1893-accN. The amplified fragment was then cut with PstI and XbaI and cloned into the PstI-XbaI site of pHN1234 (Nakashima and Tamura 2012), yielding pHN2125. A DNA fragment containing Ptet and transcriptional regulator gene (tet $R$ ) was PCR-amplified using primers sSN1894-tetR and sSN1895-tetR from pHN1271 (Nakashima and Tamura 2013). That amplified fragment was cut with SpeI and BamHI and cloned into the SpeIBamHI site of pHN2125, yielding pHN2127. A DNA fragment containing the $3^{\prime}$-flanking region of $a c c B C$ was PCR-amplified using primers sSN1896-accORF and sSN1897-accORF. The amplified fragment was then cut with $\mathrm{BamHI}$ and $\mathrm{NcoI}$ and cloned into the $\mathrm{BamHI}-\mathrm{NcoI}$ site of pHN2127, yielding pHN2128. Plasmid pHN2128 was used to knock in Ptet and tetR into the upstream of accBC.

To introduce Ptet upstream of aceE within the genome, pHN2198 was constructed. A DNA fragment containing the $5^{\prime}$-flanking region of aceE was PCR-amplified using primers sSN1997aceEDox1 and sSN1998aceEDox2. The amplified fragment was then cut with PstI and $X b a \mathrm{I}$ and cloned into the PstI-XbaI site of pHN1234 (Nakashima and Tamura 2012), yielding pHN2187. A DNA fragment containing Ptet was PCR-amplified using primers sSN1998aceEDox3 and sSN1999aceEDox4, with pHN1271 serving as a template. The amplified fragment was then cut with SpeI and PstI and ligated into the SpeI-BamHI site of pBluScriptII KS(+) using T4 DNA ligase, yielding pHN2189. A DNA fragment containing the $3^{\prime}$-flanking region of aceE was PCR-amplified using primers sSN2000aceEDox5 and sSN2001aceEDox6, after which the amplified fragment was cut with Pst $\mathrm{I}$ and EcoRV and cloned into the PstI-EcoRV site of pHN2189, yielding pHN2191. A DNA fragment cut from pHN2191 using SpeI and NcoI was cloned into the $\mathrm{XbaI}-\mathrm{NcoI}$ site of pHN2187, yielding pHN2198.

\section{Standard culture conditions for pyruvate production}

Strain LAFCPCPt-accBC-aceE was pre-grown overnight in Luria-Bertani medium and then diluted 1:100 with fresh N5G medium (pH 7.2) containing $60 \mathrm{~g} \mathrm{~L}^{-1}$ glucose, $10 \mathrm{~g} \mathrm{~L}^{-1}\left(\mathrm{NH}_{4}\right)_{2} \mathrm{SO}_{4}, 2 \mathrm{~g} \mathrm{~L}^{-1} \mathrm{NaCl}, 1 \mathrm{~g} \mathrm{~L}^{-1} \mathrm{KH}_{2} \mathrm{PO}_{4}$, $0.24 \mathrm{~g} \mathrm{~L}^{-1} \mathrm{MgSO}_{4} \cdot 7 \mathrm{H}_{2} \mathrm{O}$ and $0.011 \mathrm{~g} \mathrm{~L}^{-1} \mathrm{CaCl}_{2} \cdot 2 \mathrm{H}_{2} \mathrm{O}$. Pyruvate production was performed in a small-scale 
multistage fermentor Bio Jr. 8 (Able \&amp; Biott, Tokyo, Japan) with a working volume of $80 \mathrm{~mL}$. The standard culture conditions were as follows: culture temperature, $37^{\circ} \mathrm{C}$; culture $\mathrm{pH}, 7.2$; stirrer speed, $1200 \mathrm{rpm}$; airflow rate, $50 \mathrm{~mL} \mathrm{~min}^{-1}$.

\section{Optimization of the culture conditions}

The effect of culture temperature was examined at 29-37 ${ }^{\circ} \mathrm{C}$. The culture $\mathrm{pH}$ was tested at $\mathrm{pH}$ 5.4-7.2 through automatic addition of $4 \mathrm{M} \mathrm{NaOH}$ solution. The stirrer speed and airflow rate were examined at 1200 $2000 \mathrm{rpm}$ and $50-150 \mathrm{~mL} \mathrm{~min}^{-1}$, respectively. The $\mathrm{OD}_{600}$ was measured by monitoring the difference between the cell and cell-free turbidity values using an Eppendorf BioSpectrometer (Eppendorf, Hamburg, Germany).

\section{Quantification of pyruvate and glucose}

After clarifying the culture by centrifugation and filtration, pyruvate and glucose were quantified using a high performance liquid chromatograph equipped with a Jasco UV-2070 Plus Intelligent UV/VIS Detector at $210 \mathrm{~nm}$ (Jasco, Tokyo, Japan), a Jasco RI-2031 Plus Intelligent Refractive Index Detector (Jasco) and an Aminex HPX87 $\mathrm{H}$ cationic exchange column connected to an Aminex 85H Micro-Guard Column (Bio-Rad Labs, Hercules, CA, USA). The chromatographic conditions were as follows: mobile phase, $4 \mathrm{mM} \mathrm{H}_{2} \mathrm{SO}_{4}$; flow rate, $0.5 \mathrm{~mL} \mathrm{~min}^{-1}$; column oven temperature, $65^{\circ} \mathrm{C}$.

\section{Results}

Control of $a c c B C$ expressions by the tetracycline-regulated promoter

In $E$. coli cells, the accABCD genes are annotated as essential genes for growth. The accABCD gene products comprise the acetyl-CoA carboxylase complex, which catalyzes the biotin-dependent carboxylation of acetyl-CoA to produce malonyl-CoA via two half-reactions (Broussard et al. 2013). In the first half-reaction, biotin carboxylase, which is encoded by $a c c C$, catalyzes the ATP-dependent carboxylation of biotin. Immediately after the carboxylation, the carboxylated biotin is attached to the biotin carboxyl carrier protein, which is encoded by $a c c B$, resulting in the synthesis of carboxybiotin. In the second half-reaction, carboxyltransferase, which is encoded by $a c c A D$, transfers the carboxyl group from carboxy-biotin to acetyl-CoA, yielding malonylCoA. In an earlier study, we observed that silencing aceE expression increased the accumulation of pyruvate (Nakashima et al. 2014b). In addition, we also reported that silencing both $a c c A$ and $a c e E$ led to even greater pyruvate accumulation (Nakashima et al. 2014b). Those results indicate that pyruvate accumulation is achieved by significantly decreasing the activities of both the pyruvate dehydrogenase complex and the acetyl-CoA carboxylase complex. In the present study, therefore, we used Ptet, which is controlled by the presence or absence of doxycycline, to regulate the expressions of $a c c B C$ and aceE.

To confirm the effect of Ptet on gene expression, we assessed our ability to control $a c c B C$ expressions. When doxycycline was present in the culture, the growth curves obtained with strain Pt-accBC were similar to those obtained with the wild-type strain (Fig. 2). On the other hand, growth of strain Pt-accBC was retarded in the absence of doxycycline (Fig. 2b). These results demonstrate that Ptet is useful for controlling essential gene expression.

\section{Development of a pyruvate producing strain}

In $E$. coli cells, acetate is produced via two primary routes (Fig. 1): (1) conversion of acetyl-CoA to acetate by phosphotransacetylase $(p t a)$ and acetate kinase (ack $A$ ) (Kakuda et al. 1994) and (2) oxidation of pyruvate to acetate by pyruvate oxidase (poxB) (Abdel-Hamid et al. 2001). To block acetate production from pyruvate in $E$. coli cells, the ackA-pta and poxB genes were deleted.

The Cra protein, global transcriptional regulator, controls transcriptional expression of genes involved in sugar catabolism (Saier and Ramseier 1996). Moreover, we previously reported that disruption of cra was also beneficial for accumulating pyruvate in $E$. coli cells, since this disruption led to a more rapid rate of pyruvate production (Nakashima et al. 2014b). Therefore, $\mathrm{cra}$ was also deleted. The resultant strain, LAFCPCPt-accBC-aceE, was then used for experimentation.

\section{Pyruvate production using standard and optimized conditions}

When $60 \mathrm{~g} \mathrm{~L}^{-1}$ glucose was provided as the carbon source under the standard culture conditions (see "Materials and methods" section), strain LAFCPCPt-accBCaceE pyruvate levels reached $4.2 \mathrm{~g} \mathrm{~L}^{-1}$ in $72 \mathrm{~h}$ with consumption of $40.5 \mathrm{~g} \mathrm{~L}^{-1}$ glucose (Fig. 3).

To optimize the culture conditions for pyruvate production by strain LAFCPCPt-accBC-aceE, the effects of temperature, initial culture $\mathrm{pH}$, stirrer speed and airflow rate were evaluated. The maximum pyruvate concentration was obtained at $35{ }^{\circ} \mathrm{C}$ (Fig. 4a). When the effect of initial culture $\mathrm{pH}$ was assessed, the highest product concentration was obtained at pH 5.7 (Fig. 4b). When the stirrer speed was investigated, the highest final product concentration was obtained at $1800 \mathrm{rpm}$ (Fig. 4c). Finally, examination of the airflow rate conditions showed that $150 \mathrm{~mL} \mathrm{~min}{ }^{-1}$ produced the highest product concentration (Fig. 4d). In sum, the optimal culture conditions for pyruvate production were as follows; 

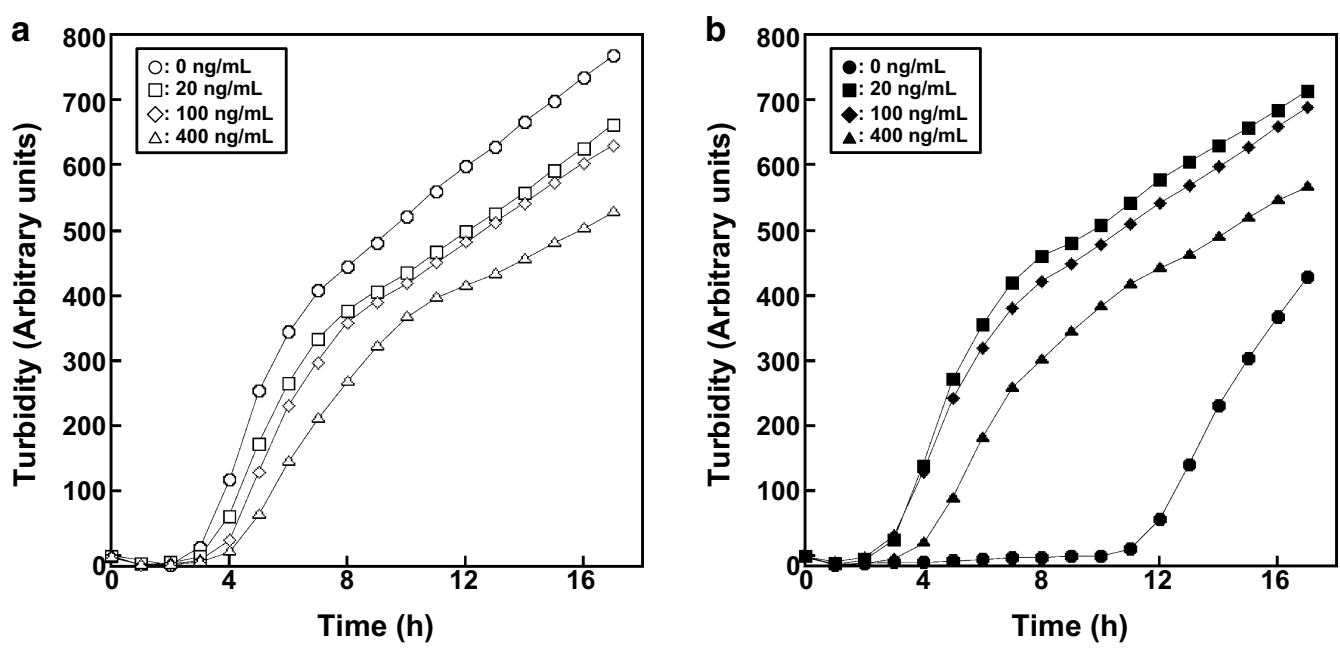

Fig. 2 Monitoring growth of wild type (a) and E. coli strain Pt-accBC (b). E. coli strains were incubated at $37^{\circ} \mathrm{C}$ in Luria-Bertani medium containing doxycycline at concentrations of $0,20,100$ and $400 \mathrm{ng} \mathrm{mL}^{-1}$, as indicated

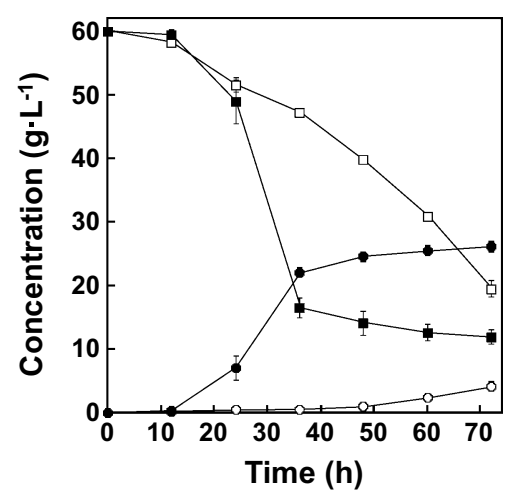

Fig. 3 Pyruvate production under standard and optimized culture conditions. The strains were cultured in N5G medium. Shown are the time-dependent changes in pyruvate (circles) and glucose (squares) concentrations under standard (open symbo/s) and optimized (filled symbols) culture conditions. Error bars indicate SE $(n=3)$

culture temperature, $35^{\circ} \mathrm{C}$; culture $\mathrm{pH}, 5.7$; stirrer speed, $1800 \mathrm{rpm}$; airflow rate, $150 \mathrm{~mL} \mathrm{~min}^{-1}$. Under these optimized conditions, the pyruvate concentration reached $26.1 \mathrm{~g} \mathrm{~L}^{-1}$ after $72 \mathrm{~h}$ with consumption of $48.0 \mathrm{~g} \mathrm{~L}^{-1}$ glucose (Fig. 3). Moreover, the productivity was 6.2-fold higher than was achieved under the standard conditions, and the theoretical yield was 5.2-fold higher. Also, strain LAFCPCPt-accBC-aceE showed improved growth rate under optimized culture conditions (Fig. 5).

\section{Discussion}

In this study, we demonstrated that Ptet was useful for controlling essential gene expressions. Subsequently, we have constructed a high pyruvate producing strain
LAFCPCPt-accBC-aceE. After optimal culture conditions for pyruvate production were determined, the final pyruvate concentration reached $26.1 \mathrm{~g} \mathrm{~L}^{-1}$ after $72 \mathrm{~h}$ with a theoretical yield of $55.6 \%$. In our knowledge, this yield was similar compared with the yields of another engineered E. coli strains (Table 1). However, our pyruvate producing strain was not required supplemental carbon additives and special expertise for pyruvate production, which was easy to perform. These results indicate that strain LAFCPCPt-accBC-aceE also has an industrial potential for pyruvate production.

With pyruvate production using strain LAFCPCPtaccBC-aceE, we think the culture $\mathrm{pH}$ is the most important factor. $\mathrm{pH}$ is known to have a significant effect on gene expression in $E$. coli cells, and expression of aceE is promoted at acidic $\mathrm{pH}$ (Maurer et al. 2005). On the other hand, after deletion of the $a c k A-p t a$ pathway, cell growth is accelerated at acidic $\mathrm{pH}$ and the activity of the $\operatorname{pox} B$ gene product is enhanced (Dittrich et al. 2005). Similarly, pyruvate productivity in a poxB-deleted strain is higher at acidic $\mathrm{pH}$ than neutral $\mathrm{pH}$ (Dittrich et al. 2005). Taken together, these observations suggest that both the production and degradation of pyruvate is stimulated at acidic $\mathrm{pH}$, and thus $\mathrm{pH} 5.7$ was optimal in our study. Note that pyruvate degradation is limited in our study due to gene manipulation.

We also observed that both stirrer speed and airflow rate influenced pyruvate production. Transcription of several genes involved in the gluconeogenesis and anaplerosis pathways ( $p c k A, p p s A, p p c$ and $s f c A$ ), the TCA cycle ( $g l t A)$, the glyoxylate cycle (aceA), and acetate metabolic pathways (acs, ackA, pta and poxB) are affected by the dissolved oxygen concentration (Phue and Shiloach 

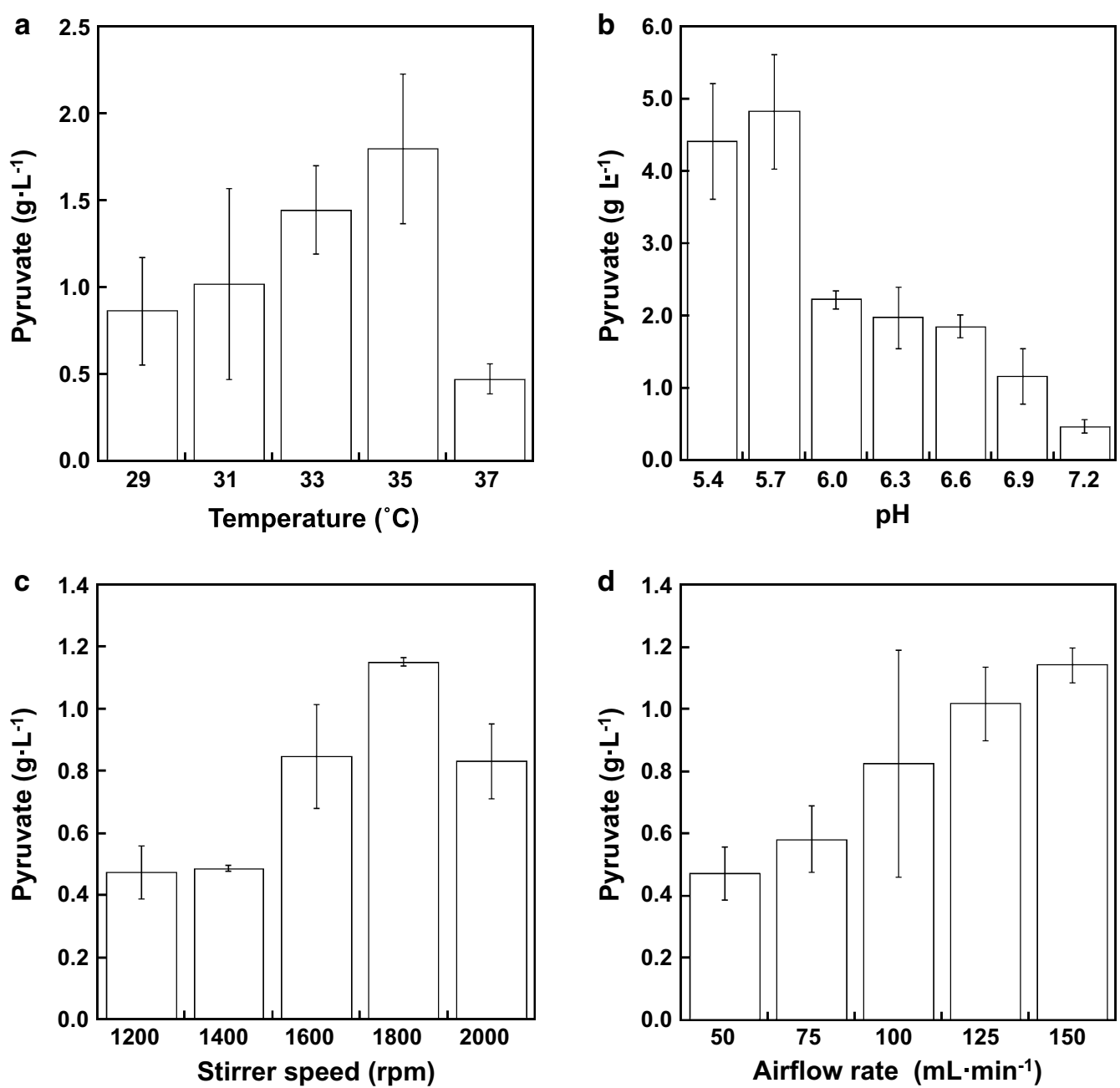

Fig. 4 Effects of culture conditions on pyruvate production. E. coli strain LAFCPCPt-accBC-aceE was cultivated for $24 \mathrm{~h}$ in N5G medium. Shown are the effects of temperature $(\mathbf{a})$, culture $\mathrm{pH}(\mathbf{b})$, stirrer speed $(\mathbf{c})$, and airflow rate $(\mathbf{d})$ on pyruvate production. Error bars indicate $\mathrm{SE}(n=3)$

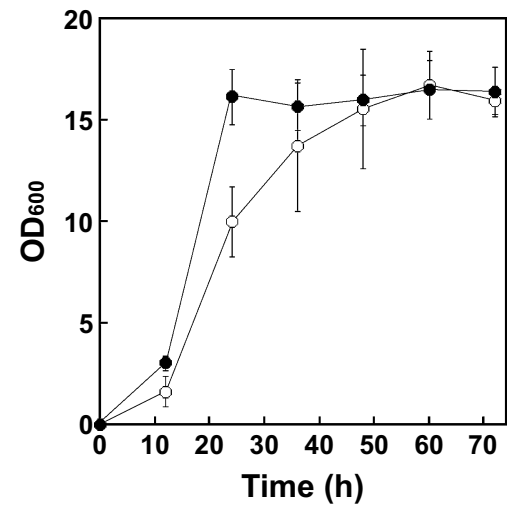

Fig. 5 Growth of E. coli strain LAFCPCPt-accBC-aceE under standard and optimized culture conditions. The strains were cultured in N5G medium under standard (open symbols) and optimized (filled symbols) culture conditions. Error bars indicate SE $(n=3)$
2005). In our study, more aerobic conditions showed improved growth rate (Fig. 5), presumably to support facilitate overall carbon flux (Matsuoka and Shimizu 2013).

We found that the optimal culture temperature was $35^{\circ} \mathrm{C}$ (Fig. 3a), though the reason is unclear. This temperature has no effect on expression of the genes involved in glycolysis or the pentose phosphate pathway (Gadgil et al. 2005). Perhaps this temperature contributes to enhancing the growth rate. For example, when E. coli strain ML30G was cultured in glucose minimal medium at various temperatures, the maximum growth rate was observed at $35^{\circ} \mathrm{C}$ (Shehata and Marr 1975).

Abbreviations

Ptet: tetracycline-regulated promoter; tetR: transcriptional regulator gene. 


\section{Authors' contributions}

HA designed this study, performed experiments, participated in the interpretation of the results and drafted the manuscript. NN participated in the design and coordination of this study and helped to revise the manuscript. TH conceived and designed this study, coordinated the experiments, interpreted the results and revised the manuscript for important intellectual content. All authors read and approved the final manuscript.

\section{Author details}

${ }^{1}$ Research Institute for Sustainable Chemistry, National Institute of Advanced Industrial Science and Technology (AIST), 3-11-32 Kagamiyama, Higashi-Hiroshima, Hiroshima 739-0046, Japan. ${ }^{2}$ Bioproduction Research Institute, National Institute of Advanced Industrial Science and Technology (AIST), 2-17-2-1 Tsukisamu-Higashi, Toyohira-ku, Sapporo 062-8517, Japan. ${ }^{3}$ Department of Biological Information, Graduate School of Bioscience and Biotechnology, Tokyo Institute of Technology, 2-12-1-M6-5 Ookayama, Meguro-ku, Tokyo 152-8550, Japan.

\section{Acknowledgements}

We are grateful to all members of the Bio-conversion Research Group at our Institute [Research Institute for Sustainable Chemistry, National Institute of Advanced Industrial Science and Technology (AIST)] for their technical assistance and valuable discussion.

\section{Competing interests}

The authors declare that they have no competing interests.

\section{Ethical approval}

This article does not contain any studies with human participants or animals performed by any of the authors.

Received: 5 July 2016 Accepted: 28 September 2016

Published online: 08 October 2016

\section{References}

Abdel-Hamid AM, Attwood MM, Guest JR (2001) Pyruvate oxidase contributes to the aerobic growth efficiency of Escherichia coli. Microbiology 147:1483-1498

Broussard TC, Price AE, Laborde SM, Waldrop GL (2013) Complex formation and regulation of Escherichia coli acetyl-CoA carboxylase. Biochemistry 52:3346-3357

CaJacob CA, Gavino GR, Frey PA (1985) Pyruvate dehydrogenase complex of Escherichia coli. Thiamin pyrophosphate and NADH-dependent hydrolysis of acetyl-CoA. J Biol Chem 260:14610-14615

Causey TB, Shanmugam KT, Yomano LP, Ingram LO (2004) Engineering Escherichia coli for efficient conversion of glucose to pyruvate. Proc Natl Acad Sci USA 101:2235-2240

Dittrich CR, Bennett GN, San KY (2005) Characterization of the acetate-producing pathways in Escherichia coli. Biotechnol Prog 21:1062-1067

EFSA (2009) Calcium acetate, calcium pyruvate, calcium succinate, magnesium pyruvate magnesium succinate and potassium malate added for nutritional purposes to food supplements. EFSA J 1088:1-25

Emmerson JR, Gally DL, Roe AJ (2006) Generation of gene deletions and gene replacements in Escherichia coli O157:H7 using a temperature sensitive allelic exchange system. Biol Proced Online 8:153-162

Gadgil M, Kapur V, Hu WS (2005) Transcriptional response of Escherichia coli to temperature shift. Biotechnol Prog 21:689-699

Kakuda H, Shiroishi K, Hosono K, Ichihara S (1994) Construction of Pta-Ack pathway deletion mutants of Escherichia coli and characteristic growth profiles of the mutants in a rich medium. Biosci Biotechnol Biochem 58:2232-2235

Kalman D, Colker CM, Wilets I, Roufs JB, Antonio J (1999) The effects of pyruvate supplementation on body composition in overweight individuals. Nutrition 15:337-340
Li Y, Chen J, Lun SY (2001a) Biotechnological production of pyruvic acid. Appl Microbiol Biotechnol 57:451-459

Li Y, Chen J, Lun SY, Rui XS (2001 b) Efficient pyruvate production by a multivitamin auxotroph of Torulopsis glabrata: key role and optimization of vitamin levels. Appl Microbiol Biotechnol 55:680-685

Matsuoka Y, Shimizu K (2013) Metabolic regulation of Escherichia coli cultivated under anaerobic and aerobic conditions in response to the specific pathway gene knockouts. Adv Biosci Biotechnol 4:455-468

Maurer LM, Yohannes E, Bondurant SS, Radmacher M, Slonczewski JL (2005) $\mathrm{pH}$ regulates genes for flagellar motility, catabolism, and oxidative stress in Escherichia coli K-12. J Bacteriol 187:304-319

Nakashima N, Miyazaki K (2014) Bacterial cellular engineering by genome editing and gene silencing. Int J Mol Sci 15:2773-2793

Nakashima N, Tamura T (2012) A new carbon catabolite repression mutation of Escherichia coli, $m / c^{*}$, and its use for producing isobutanol. J Biosci Bioeng 114:38-44

Nakashima N, Tamura T (2013) Gene silencing in Escherichia coli using antisense RNAs expressed from doxycycline-inducible vectors. Lett Appl Microbiol 56:436-442

Nakashima N, Akita H, Hoshino T (2014a) Establishment of a novel gene expression method, BICES (biomass-inducible chromosome-based expression system), and its application for production of 2,3-butanedio and acetoin. Metab Eng 25:204-214

Nakashima N, Ohno S, Yoshikawa K, Shimizu H, Tamura T (2014b) A vector library for silencing central carbon metabolism genes with antisense RNAs in Escherichia coli. Appl Environ Microbiol 80:564-573

Park HS, Lee JY, Kim HS (1998) Production of L-DOPA(3,4-dihydroxyphenylL-alanine) from benzene by using a hybrid pathway. Biotechnol Bioeng 58:339-343

Phue JN, Shiloach J (2005) Impact of dissolved oxygen concentration on acetate accumulation and physiology of E. coli BL21, evaluating transcription levels of key genes at different dissolved oxygen conditions. Metab Eng 7:353-363

Rosche B, Sandford V, Breuer M, Hauer B, Rogers P (2001) Biotransformation of benzaldehyde into $(R)$-phenylacetylcarbinol by filamentous fungi or their extracts. Appl Microbiol Biotechnol 57:309-315

Saier MH Jr, Ramseier TM (1996) The catabolite repressor/activator (Cra) protein of enteric bacteria. J Bacteriol 178:3411-3417

Saper RB, Eisenberg DM, Phillips RS (2004) Common dietary supplements for weight loss. Am Fam Physician 70:1731-1738

Shehata TE, Marr AG (1975) Effect of temperature on the size of Escherichia coli cells. J Bacteriol 124:857-862

Tomar A, Eiteman MA, Altman E (2003) The effect of acetate pathway mutations on the production of pyruvate in Escherichia coli. Appl Microbiol Biotechnol 62:76-82

Wang X, Perez E, Liu R, Yan LJ, Mallet RT, Yang SH (2007) Pyruvate protects mitochondria from oxidative stress in human neuroblastoma. SK-N-SH cells. Brain Res 1132:1-9

Xu P, Qiu J, Gao C, Ma C (2008) Biotechnological routes to pyruvate production. J Biosci Bioeng 105:169-175

Yokota A, Terasawa Y, Takaoka N, Shimizu H, Tomita F (1994) Pyruvic acid production by an $\mathrm{F}_{1}$-ATPase-defective mutant of Escherichia coli W1485lip2. Biosci Biotechnol Biochem 58:2164-2167

Zelić B, Gerharz T, Bott D, Vasić-Racki D, Wandrey C, Takors R (2003) Fed-batch process for pyruvate production by recombinant Escherichia coli YYC 202 strain. Eng Life Sci 3:299-305

Zhang Y, Tao F, Du M, Ma C, Oiu J, Gu L, He X, Xu P (2010) An efficient method for $\mathrm{N}$-acetyl-D-neuraminic acid production using coupled bacterial cells with a safe temperature-induced system. Appl Microbiol Biotechnol 86:481-489

Zhu Y, Eiteman MA, Altman R, Altman E (2008) High glycolytic flux improves pyruvate production by a metabolically engineered Escherichia coli strain. Appl Environ Microbiol 74:6649-6655 